\title{
Hábitos ecológicos y conservación del medio ambiente en estudiantes de primaria
}

\author{
Rossiell María Urbina Lozano \\ urbinarossiel120@gmail.com \\ Orcid: https://orcid.org/0000-0002-5494-5515 \\ Afiliación institucional del equipo de investigación \\ Universidad Cesar Vallejo \\ Trujillo - Perú
}

\section{RESUMEN}

La presente investigación tuvo como objetivo determinar la relación de los hábitos ecológicos y la conservación del medio ambiente de los estudiantes del tercer grado del nivel primaria de la institución educativa No 81011 de Trujillo en el año 2020, en un estudio correlacional causal. La población estuvo constituida por 161 estudiantes, siendo la muestra 53. Como instrumentos, se utilizó el Test de hábitos ecológicos y el test de conservación del medio ambiente. Los resultados en hábitos ecológicos, mostraron que el $77,4 \%$ de los estudiantes se encuentran en el nivel de logrado, el 22,6\% en proceso y el $8 \%$ en inicio, mientras que, en la conservación del medio ambiente el 77,4\% se halla en el nivel de logrado, el 22,6\% en proceso y el $8 \%$ en inicio. Asimismo, se evidencia que no existe correlación en el estadístico general entre los hábitos ecológicos y la conservación del medio ambiente, pues la prueba estadística rho de Spearman arroja un valor $\mathrm{r}$ de -,089 y un nivel de significancia p de 0,526, sin embargo, entre las dimensiones de las variables existe significatividad en el reciclaje de residuos sólidos con las dimensiones cognitiva y activa y la limpieza del aula con la dimensión conativa.

Palabras claves: cognitivo; afectivo; conativa; reciclaje; residuos sólidos. 


\title{
Ecological habits and environmental conservation in \\ elementary school students
}

\begin{abstract}
The objective of this research was to determine the relationship of ecological habits and environmental conservation of third grade students of the primary level of the educational institution No. 81011 of Trujillo in 2020, in a causal correlational study. The population consisted of 161 students, the sample being 53. As instruments, the ecological habits test and the environmental conservation test were used. The results in ecological habits showed that $77.4 \%$ of the students are at the achieved level, $22.6 \%$ in process and $8 \%$ in the beginning, while, in the conservation of the environment, $77,4 \%$ is at the level of achieved, $22.6 \%$ in process and $8 \%$ in start. Likewise, it is evidenced that there is no correlation in the general statistic between ecological habits and environmental conservation, since Spearman's rho statistical test yields an $\mathrm{r}$ value of -. 089 and a significance level $\mathrm{p}$ of 0.526 , however between In the dimensions of the variables, there is significance in the recycling of solid waste with the cognitive and active dimensions and the cleanliness of the classroom with the conative dimension.
\end{abstract}

Keywords: cognitive; affective; conative; recycling; solid waste.

Artículo recibido: 10 Agosto. 2021 Aceptado para publicación: 07. Setiembre. 2021 Correspondencia: urbinarossiell20@gmail.com Conflictos de Interés: Ninguna que declarar 


\section{INTRODUCCIÓN}

La humanidad ve con preocupación cómo se está deteriorando el ambiente, lo que perjudica notablemente la salud del ser humano. La salud, la educación y el desarrollo guardan una relación directa; una comunidad debe garantizar condiciones básicas para contar con un ambiente seguro como alimentos sanos, agua pura, y ambientes adecuados en las instituciones educativas.

Páramo y Gómez (1997) han estimado que el escaso involucramiento de los individuos ante el problema ambiental es producto de que el medio en que vivimos no está incluido en los conocimientos simultáneos que tenemos de nuestro ambiente.

En el Perú, los problemas de contaminación ambiental se incrementan cada día, debido mayormente a la falta de una conciencia ambiental, a la falta de práctica de hábitos ecológicos y a la interacción de la sociedad con la naturaleza. En este sentido lo que produce una inadecuada práctica de los hábitos ecológicos para la conservación del medio ambiente, no es la inexistencia de normas que fomenten su enseñanza en el contexto educativo o social, sino a la desarticulación de los conocimientos para una evaluación interdisciplinaria de la situación y a la separación de los sistemas educativos de la evaluación y solución del problema ambiental, desde un punto de vista ecológico y moral. Del mismo modo, la región La Libertad debido al progreso de la tecnología y la escasa práctica de valores de las personas se han incrementado problemas de la formación de hábito ecológico y de preservación del medio ambiente, podemos observar la alteración del paisaje, deforestación, reducción de la biodiversidad, contaminación fluvial por basura, aguas residuales de uso doméstico y mineros, con el consecuente aporte al calentamiento del planeta y al efecto invernadero.

La dificultad se explica por la exigua experiencia de los docentes en la vinculación de los instrumentos de administración educativa, además, la actividad educativa está dirigida concretamente a los estudiantes, no considerando la intervención de los otros componentes de la comunidad educativa como son el docente y los padres de familia. También se evidencia la escasa contribución de los organismos públicos e instituciones estatales y privadas de la localidad en las acciones pedagógicas de nuestra entidad.

En lo referente a la labor con los estudiantes, en la institución educativa se ha detectado que un elevado porcentaje de niños que arrojan las envolturas de golosinas, papeles y toda clase de residuos sólidos en el piso, simplemente no existe una cultura ecológica. Los 
docentes consideran que se debe a la falta de estimulación temprana e indiferencia de los tutores, a la carencia de normas en la entidad y a la falta de experiencias y procedimientos en la adquisición y fomento de buenos hábitos ecológicos.

Sin embargo, cuando los docentes programan actividades de aseo y limpieza colectiva de los ambientes del centro educativo, abandonan al estudiante a su suerte o les exigen que realicen las labores de higiene ambiental sin ningún criterio. Muy pocas veces encontramos que los agentes educativos, en su conjunto, asuman su responsabilidad en este gran problema, que se agudiza y se encuentra quizá más generalizado que en décadas anteriores.

De manera específica se puede apreciar esta situación problemática en los estudiantes del tercer grado, quienes presentan dificultades para conservar el ambiente limpio, además demuestran escasa cultura de salubridad, se evidencia en ellos, la falta de sensibilización para la práctica de los hábitos ecológicos adecuados. Además, los niños no toman conciencia del riesgo que corre su salud al desarrollar sus clases dentro de ambientes que no están debidamente aseados.

Teniendo en cuenta que el hábito ecológico y el amor hacia la preservación del ambiente de los alumnos del nivel primario debe fortalecerse, se propone determinar la influencia de los hábitos ecológicos en la preservación del medio ambiente en los educandos del tercer grado de primaria de la IE “Antonio Raimondi” de Trujillo, con el fin de transmitir el resultado y desplegar tácticas encaminadas a otorgar al docente conocimientos sobre el nivel en que se hallan los alumnos en las dimensiones activa, conativa afectiva y cognitiva e incrementar sus conocimientos sobre el problema ambiental, además de brindar la base para crear proyectos sostenibles para mejorar el ambiente escolar.

\section{En consecuencia, el problema de investigación es:}

- ¿En qué medida los hábitos ecológicos influyen en la conservación del medio ambiente en los alumnos del tercer grado de educación primaria de la IE N 81011 “Antonio Raimondi” de Trujillo, 2020?

\section{En concordancia con el problema el Objetivo General fue:}

- Determinar la influencia de los hábitos ecológicos en la conservación del medio ambiente de los alumnos del tercer grado de nivel primario de la IE No 81011 “Antonio Raimondi” de Trujillo en el año 2020. 


\section{Y los Objetivos Específicos:}

- Identificar el nivel actual de la práctica de hábitos ecológicos de los estudiantes del 3er. Grado. Identificar el nivel de conservación del medio ambiente de los estudiantes del tercer grado de primaria.

- Determinar la influencia de los hábitos ecológicos en la conservación del medio ambiente en los alumnos del tercer grado de primaria, en el año 2020.

- Determinar la influencia de las dimensiones de la variable hábitos ecológicos en las dimensiones de la variable conservación del medio ambiente en los alumnos del tercer grado de primaria, en el año 2020.

\section{Referente Teórico}

Desde la perspectiva teórica, el presente estudio hábitos ecológicos y su influencia en la conservación del medio ambiente tiene su fundamento científico en el Paradigma Sociocultural, que según Saegert y Winkel (1990), sostienen que el comportamiento humano o llamado conducta es un acto social, el mismo que depende de las circunstancias socioculturales y como estas se relacionan en un tiempo y espacio determinados.

El enfoque sociocultural destaca el carácter del individuo como agente social, también el dominio figurado de las interacciones sociales, en relación a considerar a la persona autónoma con necesidad de supervivencia y planes personales. La persona como agente social busca crear sentido a partir del entorno. Entonces el comportamiento hacia el cuidado ambiental varía según el ambiente en que se encuentra la persona: en un espacio privado (dormitorio), espacio público (escuela, centro comercial).

En referencia a las dimensiones de los Hábitos ecológicos, Morejón (2006), menciona que la primera dimensión es el cuidado de las áreas verdes del centro educativo, allí se encuentran las plantas ornamentales, el césped y los árboles que ayudan a purificar el aire de nuestro entorno, embellece el ornato y según Gareca y Villarpando (2017), afirman que estas zonas especiales de los centros educativos son significativos para el progreso de los estudiantes principalmente en los aspectos intelectual, emocional, social, espiritual y físico. Asimismo, asegura que el contacto con la naturaleza redunda en forma positiva en el modo de vida de los infantes.

La segunda dimensión es el reciclaje de residuos sólidos, la cual busca desarrollar el uso correcto de contenedores para un uso posterior como materia prima de otros productos. 
El 90\% de los residuos sólidos se vuelven utilizar proporcionando que el estudiante pueda hacer uso de su creatividad, elaborando otros productos.

La tercera dimensión es limpieza del aula, que consiste en conservar los ambientes limpios y apropiados para los estudiantes y a los profesores para educar, para lograr esto todos debemos participar, tratando de ensuciar menos conservaremos nuestra salud y bienestar. Allí radica la importancia por cuanto, el aula se convierte en un ambiente saludable y aseado para aprender y para convivir con nuestros compañeros. Las aulas sucias entorpecen el aprendizaje por el mal aspecto de los desperdicios, polvo, etc. Produciendo un bajo rendimiento de los estudiantes y propician la adquisición de enfermedades.

En relación a la segunda variable, conservación del medio ambiente, se fundamenta en el enfoque cognitivo evolutista o constructivista, que igualmente se ha denominado “orientación internalista de la moral”, que afirma que el desarrollo de la noción de preservación del medio ambiente en los estudiantes expresa la reconstrucción gradual por parte del educando de un sentido moralista, que le dirige a una deliberación y entendimiento maduro de las reglas y valores y se basa específicamente en la educación ambiental.

En este sentido, educar para conservar el medio ambiente, según Fernández (1992), es el procedimiento de identificación de los valores y aclarar ideas para establecer las destrezas y cualidades que se necesitan, para entender y conocer las interrelaciones entre la persona, su conocimiento y el ambiente físico que lo envuelve. Vílchez (2003), por su parte afirma que educar para conservar el medio ambiente es un enfoque universal de ideología y práctica. El fin es promover un cambio individual y social para provocar la mejoría del ambiente y un progreso sustentable.

En consecuencia, la educación para la conservación del medio ambiente es una parte de la formación que busca mayor eficiencia social e intenta ser impulsor del desarrollo de las comunidades, buscando la conservación de las características del entorno físico y de la buena condición de vida. Es instruir el amor hacia todo lo natural y la cultura humana, la concienciación de dependencia entre todos los seres vivientes y el ecosistema en que habitan, y la obligación de participación solidaria.

De igual manera, en el presente trabajo, se considera la Teoría cognitiva propuesta por la psicología ambiental, con el Modelo de Activación de las Normas Morales por cuanto 
según Lehman (2004), los comportamientos permanentes se relacionan con los que poseen características prosociales o morales que tiene todo ser humano; puesto que el comportamiento prosocial o filántropo se define como aquel que, siendo propio de la persona, origina beneficios en provecho de la sociedad.

En este sentido, Barker (1968), afirma que el enfoque estudia la manera en que el estudiante observa y precisa un acontecimiento que demanda revolverse por medio de una decisión moral, en nuestra investigación, sobre que conducta debe tener para conservar el medio ambiente.

Se activa la regla moral cuando se presentan dos estimaciones cognitivas: a) que el individuo conozca que su comportamiento tiene resultados negativos en la prosperidad de otros seres humanos (concienciación de consecuencias); y b) que el individuo reconozca que tiene un nivel de responsabilidad si existen consecuencias negativas como resultado de sus acciones (ubicación de responsabilidad). Estos dos requerimientos son importantes para que el buen comportamiento se origine, por cuanto intervienen activando las reglas morales o personales. La aceptación de este enfoque permite explicar el comportamiento hacia el ambiente, por la preocupación que manifiestan los seres humanos sobre cómo la contaminación del medio ambiente afecta también a sus seres queridos.

Este enfoque permite establecer una correlación causal entre sus variables que permiten hacer efectivos comportamientos ecológicos responsables con el ambiente, como reciclar vidrio o las relacionadas con la eficiencia energética.

Corraliza y Berenguer (2000) aprueban el resultado de la atención de este punto de vista, reconociendo dos elementos básicos del comportamiento ambiental: El valor moral (procede de activar la regla moral individual, las emociones como resultado del deber moral y la generosidad) y los dogmas ambientales (emergen como resultado del estudio sobre costo - beneficio que el individuo efectúa sobre las consecuencias de su comportamiento).

Con respecto a las dimensiones según Corraliza (2004), se pueden distinguir cuatro para la conservación del medio ambiente: cognitiva, afectiva, conativa y activa.

Para Corraliza (2004), la dimensión cognitiva es la agrupación de nociones o que orientan los niveles de conocimientos e informaciones sobre todo lo relacionado con el medio ambiente, considerando a éste medio como un entorno diario importante que lo conduce 
a revelar su contexto como forma de vivir por intermedio de la indagación en el espacio y tiempo; sus actuales vivencias diarias, la forma como lo aprecia y lo analiza, que identifica a la propia persona dentro de la sociedad teniendo en cuenta su acervo cultural y ambiental. Aquí incluimos el conocimiento básico sobre la conservación del ambiente, la búsqueda de información pertinente para comprender los problemas y los fenómenos ambientales, la valoración de los diálogos y análisis crítico de los conocimientos para la toma de decisiones correctas, teniendo en cuenta el pasado, el presente y el futuro, para efectuar una reflexión moral.

Con respecto a la dimensión afectiva, Corraliza (2004), afirma que está referida al grupo de emociones que muestran los dogmas y el sentimiento en el tema medioambiental. En este sentido, el respeto por el medio en que vivimos no es solo un grupo de dificultades a solucionar, sino que además es una forma de existencia con la que se desarrolla una orientación de pertenecer a un determinado ambiente y crear planes y proyectos para conservarlo, como cuando valoramos lo bio-cultural o el eco desarrollo, desde un punto de vista centrado en los comportamientos morales.

Por su parte, la dimensión conativa agrupa los comportamientos que inclinan a tener una conducta crítica y a interesarse por intervenir en gestiones para contribuir a contrarrestar la problemática medioambiental. Además, incluye las acciones correspondientes a comportamientos deliberados que son éticamente fundamentados. Tal como el involucramiento en la solución de los problemas concretos y en el impulso de proyectos de mejora ambiental, formando personas competentes con el valor moral de aportar, con alto grado de reflexión para la acción. Esta dimensión es llamada también fase autoexhortativa o comportamiento moral.

Finalmente, la dimensión activa, involucra aquellos comportamientos que acarrean la ejecución de prácticas y conductas ambientales con responsabilidad, en forma individual como agrupada, inclusive en condiciones de presión. Son comportamientos éticos y comprometidos basados en el conocimiento crítico y lúcido, que enlace "el ser con el actuar", a nivel particular i/o agrupado. Educarse para convivir y para laborar unidos, en cooperación, discernir, oír, dialogar, persuadir para lograr una intervención ambientalista eficaz. 


\section{ESTRATEGIAS METODOLÓGICAS O MATERIALES Y MÉTODOS}

\subsection{Enfoque}

El presente es un estudio no experimental descriptivo, correlacional causal con método cuantitativo (Hernández, Fernández, Baptista, 2010). Orientado a determinar el nivel de relación que existe entre los hábitos ecológicos y la conservación del medio ambiente.

\subsection{Unidades de estudio}

La población estuvo constituida por 161 estudiantes de tercer grado de primaria de la institución educativa $N^{\circ} 81011$ “Antonio Raimondi” de la ciudad de Trujillo, región La Libertad, Perú, con edades comprendidas entre 7 y 8 años, del género masculino y contexto económico medio bajo, siendo la muestra 53 de ellos seleccionados mediante un muestreo aleatorio estratificado simple.

\subsection{Técnicas de recolección}

Al grupo elegido se le administró los test de hábitos ecológicos y de conservación del medio ambiente, los mismos que fueron validados por 5 expertos, doctores en educación, quienes valoraron la congruencia de las dimensiones, categorías, indicadores e ítems, conjuntamente con la redacción y claridad, luego fueron aplicados para hallar el nivel de los estudiantes en cada variable.

Para determinar la confiabilidad de los instrumentos fue necesaria la aplicación de una prueba piloto a un grupo de 10 alumnos del tercer grado que no pertenecían a la muestra, pero con tipología similar. Luego se determinó el índice con el Alfa de Cronbach que dio como resultado, 0,871 para el test de hábitos ecológicos y 0,862 para la prueba de conservación del medio ambiente, lo cual indica que son altamente confiables.

\subsection{Procesamiento de los datos}

Para el análisis de los datos se hizo uso de la estadística descriptiva y del Coeficiente de correlación de Spearman. Todo ello trabajado con el programa SPSS Statistic versión 25.

\section{RESULTADOS Y DISCUSIÓN}

Al realizar la tabulación de los datos obtenidos y con el propósito de responder a nuestros objetivos y a diversas interrogantes planteadas en el estudio: Hábitos ecológicos y conservación del medio ambiente de los alumnos del tercer grado de primaria de la 
institución educativa № 81011 “Antonio Raimondi” de Trujillo en el año 2020, luego del procesamiento de los datos (calificaciones y baremaciones) se procede a sintetizar los resultados, que se muestran a continuación.

\section{Nivel descriptivo}

Para la variable hábitos ecológicos se ha establecido tres niveles logrado, proceso y en inicio, como se presenta en la tabla siguiente.

Tabla 1: Nivel de la variable hábitos ecológicos de los estudiantes del 3er grado de la IE. $N^{\circ} 81011$ de Trujillo, 2020

\begin{tabular}{lcc}
\hline Nivel & Frecuencia & Porcentaje $\%$ \\
\hline Logrado & 41 & 77,4 \\
Proceso & 12 & 22,6 \\
Inicio & 00 & 0,0 \\
Total & $\mathbf{5 3}$ & $\mathbf{1 0 0 , 0}$ \\
\hline
\end{tabular}

Fuente: Elaboración propia, con la base de datos hábitos ecológicos

\section{Descripción}

En la tabla 1 se puede observar que la mayoría de los estudiantes valorados en la variable hábitos ecológicos, se clasifican en el nivel de logrado con 77,4\%, el 22,6\% se ubica en el nivel de proceso, y el $8 \%$ en el nivel de inicio.

Esto significa que los estudiantes evaluados se hallan en un nivel alto, no obstante, es necesario que para lograr una normalidad adecuada la docente debe esforzarse para conseguir que el $100 \%$ de alumnos alcancen el nivel de logrado en esta variable.

Tabla 2: Nivel de la variable conservación del medio ambiente de los estudiantes del 3er grado de la IE. $N^{\circ} 81011$ de Trujillo, 2020

\begin{tabular}{lcc}
\hline Nivel & Frecuencia & Porcentaje \% \\
\hline Logrado & 41 & 77,4 \\
Proceso & 12 & 22,6 \\
Inicio & 00 & 0,0 \\
Total & 53 & 100,0 \\
\hline
\end{tabular}

Fuente: Elaboración propia, con base de datos conservación del medio ambiente

\section{Descripción}

En la tabla 2 se observa que la mayoría de los estudiantes valorados en la variable conservación del medio ambiente, se clasifican en el nivel de logrado con 77,4\%, el $22,6 \%$ se ubicada en el nivel de proceso, y el $8 \%$ en el nivel de inicio. 
Esto significa que los estudiantes evaluados se hallan en un nivel aceptable, no obstante, es necesario que para lograr una regularidad adecuada la docente debe esforzarse para que el $100 \%$ de alumnos alcancen el nivel de logrado en esta variable.

Tabla 3 Pruebas de normalidad de Kolmogorov Smirnov y Shapiro Wilk

\begin{tabular}{lccccccc}
\hline & \multicolumn{3}{c}{ Kolmogorov-Smirnov $^{\text {a }}$} & \multicolumn{3}{c}{ Shapiro-Wilk } \\
VARIABLE & Estadístico & Gl & Sig. & Estadístico & gl & Sig. \\
Hábito ecológico & & 0,228 & 53 & 0,000 & 0,885 & 53 & 0,000 \\
$\begin{array}{l}\text { Conservación } \\
\text { medio ambiente }\end{array}$ & del & 0,240 & 53 & 0,000 & 0,892 & 53 & 0,000
\end{tabular}

Fuente: Análisis estadístico de datos en programa SPSS

Como el tamaño de la muestra es superior a 50 usamos los resultados de la prueba de Kolmogorov - Smimov y comprobamos que el nivel de significación, si es menor que 0.05, entonces la distribución no es normal. El resultado sugiere que la correlación de las variables evaluadas deberá ser analizada utilizando la prueba estadística no paramétrica Rho de Spearman.

Tabla 4 Tabulación cruzada de las variables hábitos ecológicos y conservación del medio ambiente, 2020

\begin{tabular}{lcccccccc} 
& & \multicolumn{3}{c}{ Habitos Ecológicos } & \multicolumn{3}{c}{ Total } \\
& NIVEL & Proceso & $\%$ & Logrado & $\%$ & N & $\%$ \\
Conservación del & Proceso & 5 & 9,4 & 7 & 13,2 & 12 & 22,6 \\
medio ambiente & Logrado & 7 & 13,2 & 34 & 64,2 & 41 & 77,4 \\
TOTAL & & 12 & 22,6 & 41 & 77,4 & 53 & 100 \\
Coeficiente de correlación &,- 089 & & Sig. (bilateral) &, 526 & & \\
\hline
\end{tabular}

Fuente: Procesamiento de datos en programa SPSS

Del total de estudiantes, $64,2 \%$ se encuentran en el nivel de logrado tanto en la variable hábitos ecológicos como en la variable conservación del medio ambiente, 13,2\% se ubican en el nivel de logrado en conservación del medio ambiente y en el nivel de proceso en hábitos ecológicos, mientras que 13,2\% de ellos se hallan en el nivel de proceso en conservación del medio ambiente y logrado en hábitos ecológicos y 9,4\% se sitúan en el nivel de proceso en ambas variables. 


\section{Contrastación de la hipótesis general:}

Hipótesis Alterna: Hi = Existe influencia directa y significativa de los hábitos ecológicos en la conservación del medio ambiente en los estudiantes del tercer grado de la IE $\mathrm{N}^{\mathrm{o}}$ 81011 “Antonio Raimondi” de Trujillo en el año 2020.

Hipótesis Nula: Ho = No existe influencia directa y significativa de los hábitos ecológicos en la conservación del medio ambiente en los estudiantes del tercer grado de la IE No 81011 “Antonio Raimondi” de Trujillo en el año 2020.

Correlación entre los hábitos ecológicos y la conservación del medio ambiente, en la Tabla 4 se visualiza que el estadístico rho de Spearman arroja un valor para $\mathrm{r}$ igual a 0,089 y un nivel de significancia p de 0,526; cómo el Valor p > 0.05, en consecuencia, se afirma que no existe influencia de los hábitos ecológicos en la conservación del medio ambiente en los estudiantes del tercer grado de primaria de la institución educativa $\mathrm{N}^{\circ}$ 81011 “Antonio Raimondi” de Trujillo en el año 2020. Por tanto, se acepta la hipótesis nula.

Tabla 5 Medidas de correlación entre las dimensiones de los hábitos ecológicos y la conservación del medio ambiente, 2020

\begin{tabular}{llcc|c}
\hline DIMENSIONES & $\begin{array}{c}\text { Cuidado de } \\
\text { las áreas } \\
\text { verdes }\end{array}$ & $\begin{array}{c}\text { Reciclaje } \\
\text { de residuos } \\
\text { sólidos }\end{array}$ & $\begin{array}{c}\text { Limpieza } \\
\text { del aula }\end{array}$ \\
\hline Cognitiva & Coeficiente de correlación & $-0,022$ & $0,313^{*}$ & $-0,154$ \\
& Sig. (bilateral) & 0,877 & 0,022 & 0,270 \\
Afectiva & Coeficiente de correlación & 0,218 & 0,227 & $-0,034$ \\
& Sig. (bilateral) & 0,117 & 0,102 & 0,810 \\
\multirow{2}{*}{ Activa } & Coeficiente de correlación & $-0,155$ & $-0,191$ & $0,307^{*}$ \\
& Sig. (bilateral) & 0,266 & 0,170 & 0,031 \\
& Coeficiente de correlación & 0,236 & $0,280^{*}$ & $-0,157$ \\
& Sig. (bilateral) & 0,090 & 0,042 & 0,262
\end{tabular}

Fuente: Procesamiento de datos en programa SPSS

Los estudios de nuestro medio ambiente, manifiestan los grandes cambios que las actividades del ser humano han producido en él, producto de un exorbitante progreso de la tecnología. Las actividades humanas entrañan muchas veces, grandes peligros que producen una transformación ecológica negativa, concretándose sus consecuencias en los diferentes elementos del ecosistema en diferente magnitud, de acuerdo a la actividad. 
Por tanto, la educación para la conservación del medio ambiente es un planteamiento que va a ayudar a menguar la contaminación del medio en una situación de vicisitudes económicas y políticas, de valores éticos, morales y de conocimientos, en un planeta sostenido por los mercados y el proceso de globalización, permitiendo la formación de una actitud crítica, las capacidades y la posibilidad de que los estudiantes adquieran conocimientos y destrezas que los lleve a valorar las dificultades de su medio para que puedan intervenir en forma activa en la solución de los mismos.

En este sentido, para conseguir el objetivo principal del estudio, se procedió a aplicar el instrumento consignado a determinar el nivel de los hábitos ecológicos de los alumnos del tercer grado del nivel primario de la institución educativa $\mathrm{N}^{\circ} 81011$ "Antonio Raimondi” de Trujillo en el año 2020.

En la tabla 1 y figura 1 se visualiza que la mayor parte de los estudiantes valorados en la variable hábitos ecológicos, se han ubicado en el nivel de logrado con 77,4\%, luego un menor porcentaje $22,6 \%$ situado en el nivel de proceso, y el $8 \%$ en el nivel de inicio. Esto significa que los estudiantes valorados se hallan en un nivel admisible, no obstante, es necesario que para instituir una regularidad beneficiosa la docente debe hacer un esfuerzo para que el 100\% de alumnos alcancen el nivel de logrado en esta variable.

Resultados similares son reportados por Larriaga (2016) en su tesis doctoral "Esquema de una Propuesta de Programa Director para la mejora de hábitos ecológicos en una institución educativa primaria” realizado en Bogotá y por Ricardes (2016), en el estudio titulado "Programa de cuidado ambientalista para el desarrollo de hábitos ecológicos en los alumnos de una Escuela Fiscal Mixta" de Quito-Ecuador, quienes afirman que hasta un $80 \%$ de los estudiantes investigados han alcanzado el nivel de logrado, desarrollando conductas y valores de cuidado ambiental contribuyendo al afianzamiento de sus culturas ambientales. Así mismo, refieren que es necesario incluir el eje transversal de la formación de hábitos ecológicos para la protección del medio ambiente en la planificación de los proyectos pedagógicos del aula e incorporarlo en el proceso de las actividades o sesiones de aprendizaje. En este sentido, se debe desplegar la conciencia ambiental, pero no viendo de manera aislada si no desde la integración de la estrategia a los documentos de gestión. Allí se establece la sostenibilidad de la misma.

En la presente investigación los resultados obtenidos del 77.4\% de estudiantes en el nivel logrado, Según Berenguer y Martín (2003), la mayoría de los alumnos tienen 
conocimiento del principio básico en el que se fundamenta la instrucción primaria, que es el rol protagónico que asume el estudiante en sus aprendizajes, por medio del proceso de asimilación, elaboración y comunicación, fundamentados en la experiencia. Este es también el principio básico sobre el que se cimienta la instrucción ambiental, privilegiando el establecimiento de valores éticos morales propensas hacia la conservación de la naturaleza, y promoviendo los cambios de actitudes instaurado en las experiencias más que en los conocimientos, cimentando los procesos educativos desde el amor hacia su entorno, en otras palabras, tiene que rescatar personalmente las experiencias que cada estudiante ha tenido en el ambiente natural en que vive.

Por otro lado, en lo referente a la variable conservación del medio ambiente, luego de realizada la evaluación se encontró, como se puede visualizar en la tabla 2 y figura 2 , que la mayor parte de los estudiantes, han alcanzado el nivel de logrado con 77,4\%, sigue un menor porcentaje $22,6 \%$ situado en el nivel de proceso, y el $8 \%$ en el nivel de inicio. Esto significa que los estudiantes valorados se hallan en un nivel admisible, no obstante, es necesario que para instituir una regularidad beneficiosa la docente debe hacer un esfuerzo para que el $100 \%$ de alumnos alcancen el nivel de logrado en esta variable.

Resultados similares son reportados por Pereda (2016), quien afirma que el 98\% de los educandos se ubicaron en el nivel de bueno, evidenciando que su estudio permitió generar conciencia ambiental desde el accionar en los espacios libres de la institución educativa, fortaleciendo la dimensión conativa y actitudinal para la conservación del medio ambiente.

Lo anterior se puede corroborar, al realizar la triangulación de los datos, como se puede visualizar en la tabla 4, donde se obtiene, que del total de estudiantes, el 64,2\% se encuentran en el nivel de logrado tanto en la variable hábitos ecológicos como en la variable conservación del medio ambiente, 13,2\% se ubican en el nivel de logrado en conservación del medio ambiente y en el nivel de proceso en hábitos ecológicos, mientras que $13,2 \%$ de ellos se encuentran en el nivel de proceso en conservación del medio ambiente y logrado en hábitos ecológicos y 9,4\% se encuentran en el nivel de proceso en ambas variables.

Sin embargo, este resultado difiere a lo manifestado por Chiape (2016), quien afirma que solo el $13 \%$ alcanza un nivel de logrado y concluye que la práctica del hábito ecológico para la preservación del medio ambiente depende de la actitud, colaboración e 
involucramiento en temas ambientales más que los conocimientos que puedan tener, pues la mayoría de los estudiantes tiene conocimiento de lo que deben hacer, pero no lo hacen, dejan los caños abiertos, arrojan desperdicios al suelo, dejan la grifería abierta, etc. Se puede evidenciar la importancia de la dimensión afectiva y conativa, las que deben ir de la mano para desarrollar hábitos ecológicos en los educandos.

Con respecto a la contratación de la hipótesis general y para evaluar la relación entre la variable hábitos ecológicos y la variable conservación del medio ambiente se utilizó el índice de Correlación rho de Spearman. Los resultados se muestran en la Tabla 4, donde se visualiza que el estadístico rho de Spearman determina una valoración para r igual a 0,089 y un nivel de significancia p de 0,526; cómo el Valor $\mathrm{p}>0.05$, se puede deducir que no existe relación entre los hábitos ecológicos y la conservación del medio ambiente en los alumnos del tercer grado de primaria de la institución educativa № 81011 “Antonio Raimondi” de Trujillo en el año 2020.

Lo cual significa que, si bien la mayoría de los estudiantes obtienen elevados porcentajes en el nivel de logrado en ambas variables, es decir poseen los conocimientos, sin embargo no muestran interés por ponerlo en práctica, lo que manifiesta que los estudiantes no se aprecian como actores importantes de contaminación y que según Márquez (1998) las alteraciones provocadas por el hombre empiezan desde que este llega al mundo y han sido permanentes, sostenidas y crecientes.

También debemos tener en cuenta los otros elementos intrínsecos, inseparables a las capacidades intelectuales y morales de los estudiantes, interés, hábito, motivación. De igual modo, hay elementos exógenos que tienen influencia como; la institución educativa, colectividad, estructura familiar, contexto social, metodologías y técnicas empleadas en las evaluaciones de las sesiones de aprendizaje, etc. Lo anterior es reconocido con Bazán y Sotero (2006) quienes manifiestan que a partiendo de los instrumentos aplicados se ha determinado que en conjunto los hábitos para la conservación del medio ambiente son más bien negativos en el país y se va incrementando con la idiosincrasia de la sociedad y con la edad.

Una prueba de ello, es cuando los estudiantes afirman que participan en acciones de conservación ambiental especialmente tratando de mantener su lugar de labores limpio, esto demuestra que el interés por su espacio laboral se ciñe a actitudes de aseo sin tener el estudiante un punto de vista adecuado que lo guíe en función de proyección al futuro. 
Por el contrario, advertimos que en los lugares donde los estudiantes realizan sus actividades es frecuente hallar papeles en el suelo y las carpetas y pupitres desordenados, lo que difiere totalmente con lo que ellos manifiestan.

En este sentido, es mínima la disposición de los estudiantes por el cuidado de su ambiente; al realizar un análisis in situ en el aula de clases de la causa de la problemática ambiental, se observa que los maestros procuran crear conocimientos que estimular su conciencia ecológica, no obstante, los comportamientos de los educandos permiten comprobar que estas actividades no son suficientes, sus actitudes de arrojar desperdicios tanto en los pasadizos de la institución educativa como en la propia aula persiste, a pesar de contar con botes de basura y contenedores en el frontis de cada aula, lo que nos conduce a realizar un juicio inaplazable de que los estudiantes deberán asumir su responsabilidad de diferente manera, reformando sus valores morales, sus conductas, sus costumbres y sus hábitos posibilitando el proceso de toma de decisiones, de tal forma que adquieran una actitud creativa y comprometida con su medio ambiente, realizando y participando en actividades concretas poniendo en práctica el desarrollo de su conocimiento ambientalista y solidario, no solo se trata de elaborar normas de comportamiento, sino de la formación en valores morales y éticos que fortalezcan, hagan comprender y transmitan las responsabilidades que toda persona tiene hacia la conservación de la naturaleza que incluye a todos los seres vivos.

Por otro lado, los resultados obtenidos al realizar la correlación entre las dimensiones de las variables en investigación como se puede observar en la tabla 5, al aplicar la prueba estadística rho de Spearman esta nos arroja que no existe correlación en todas las relaciones evaluadas, lo que nos indica que los alumnos no son participantes espontáneos en la conservación del medio ambiente, están demostrando actitudes desfavorables hacia el medio, lo que redundará negativamente en su participación en la sociedad y tiene una correlación nula con las sesiones de aprendizaje desarrolladas sobre el tema. En la conservación del medio ambiente los resultados de sus aprendizajes que manifiestan son, la oposición a las normas, negatividad, frustraciones, etc.

Esto coincide con Torres (1998) quien afirma que los hábitos ecológicos no son congénitos, sino que se presentan dependiendo de los aprendizajes, asimismo, alude que la adquisición de un hábito es producto de un proceso de formación de un concepto sobre su persona. 
Por tanto, se hace necesario concienciar a los docentes sobre la trascendencia de su tarea como forjadores de hábitos ecológicos, no solamente para la preservación de la naturaleza, sino de todas aquellas conductas que ayuden a optimizar la calidad de vida. En este sentido, se necesita de parte de los docentes una aproximación a su labor para reiterar los valores de generosidad, respeto, y solidaridad. Es necesaria la combinación de la transferencia de contenidos temáticos con la instauración de nuevas sensibilidades, enfatizar la instrucción en valores como la condescendencia, la colaboración o la solidaridad, esto debe suponer educar con el ejemplo, aportando una explícita visión de la realidad en la que vivimos. (Grupo Entorno, 2001:48).

Con respecto a la relación de la dimensión reciclaje de residuos sólidos con las dimensiones de la variable conservación del medio ambiente, se obtiene que no existe correlación en nueve de ellas, esto es debido a que los estudiantes confunden los términos reutilizar y reciclar, otorgándoles el mismo significado, por tanto, la definición de "reciclar" para los estudiantes significa lo mismo que "reutilizar", no obstante se tiene el conocimiento que reciclar puede conceptualizarse como la actividad de restituir al ciclo de utilización los objetos que han sido ya desechados y que están en condiciones de ser reutilizados para obtener otros productos. Teniendo en cuenta que la reutilización no indispensablemente requiere transformar o realizar un procesamiento nuevo, muchas veces se lava, desinfecta, adecua o modifica un producto desechado para darle otra utilidad. (Capistrán, 1998).

En este sentido, se puede deducir que los procesos educativos, en la institución educativa referida en el presente estudio, debe fomentar cambios en la comprensión de los conceptos que tienen relación con el cuidado del medio ambiente e indudablemente tener la convicción de que los profesores, en primer lugar, tienen una clara definición de los términos para no crear alguna confusión en los alumnos.

Asimismo, los estudiantes hallan una relación cercana de los vocablos reutilizar y reciclar con la idea de basura, considerando que todo cartón, papel, latas de gaseosa y plástico que ya ha sido utilizado forma parte de la basura. La manera de conceptualizar los vocablos antes mencionados, refleja ciertas conductas que se muestran diariamente en la institución educativa, puesto que pese a haberse insistido durante el desarrollo de las sesiones de aprendizaje que la disgregación de los desperdicios es una gran opción para disminuir la contaminación ambiental y del aprovechamiento máximo del recurso papel 
( uno de los materiales que en mayor cantidad se derrocha en los salones de clase) las experiencias nos indican que esta acción no se ejecuta, y la explicación se basa en que para los estudiantes todo objeto o material que se desecha es basura; pero estos comportamientos no son exclusivos de los estudiantes, sino que también de los profesores.

De manera específica, todas las cosas que ya dejaron de ser útiles o que simple y llanamente son descartados constituyen parte de la basura. Se debe hacer mención que lo que para unas personas es definida como basura, para otras personas significa un producto del cual puede sacar provecho, de tal forma que, es un asunto de cómo se conceptualice. Con respecto a los resultados de la presente investigación, debemos afirmar que a medida que los estudiantes posean las ideas claras sobre las definiciones de los términos ambientales, adquirirán una mejor competencia en la forma de estructuración lógica su manera de pensar. Por cuanto la construcción de los conocimientos son procesos en el que el estudiante realiza selección, analiza e interpreta las informaciones que recaba de diferentes fuentes y establece la relación entre esta información y su idea o conocimiento previo. (Díaz Barriga y Hernández. 2002:65).

En este sentido, la obtención de un concepto genera una serie de problemas para los estudiantes, por cuanto el desarrollo de estos conceptos es lento y muchas veces algunos de ellos sirven como sustento a otros conceptos que deberían ser transformados, lo que causa casi siempre desconcierto en los estudiantes y retardo en los aprendizajes, porque se tiene que hacer un cambio en los conceptos equivocados aprendidos.

Con todo lo expuesto no debe desalentarnos estos contextos, se debe asumir que también es posible instruirse de los errores y que los estudiantes han de poseer la virtud de percatarse de ello para que logren construir un aprendizaje propio significativo. La construcción de un conocimiento nuevo involucra un cambio en el esquema de los conocimientos que se tienen anticipadamente, esto se puede lograr instituyendo una nueva relación entre estos elementos (Díaz Barriga y Hernández. 2002:65); así de esta manera, los estudiantes pueden reformar sus ideologías para realizar una ampliación y el ajuste de su esquema de conocimiento obtenidos en los procesos de enseñanza aprendizaje.

En este sentido, al no poner en práctica los hábitos ecológicos, se incrementa el riesgo del detrimento del medio ambiente por los agentes contaminantes de diferente índole, que trae como consecuencia la muerte de todo organismo vivo y la destrucción de los 
ecosistemas. En conclusión, el problema ambiental que se vive en la era actual es el causante del deterioro de la calidad de vida, no solamente de la mayoría de los seres humanos, sino de todos los seres vivos y son en gran cuantía resultado de la no aplicación de los conocimientos sobre la conservación del medio ambiente, de la incorrecta explotación y manejo adecuado de los recursos que nos brinda la naturaleza. (Carabias, 1999:17).

Finalmente, de acuerdo a los resultados obtenidos en la presente investigación, se propone que durante los procesos educativos es sumamente importante desplegar una instrucción vicaria, en la cual las actividades docentes, por medio del modelamiento fomente los cambios de actitud valorando la trascendencia del respeto de los estudiantes hacia su persona, así como al medio ambiente que lo rodea, por ello vale destacar lo planteado por Díaz Barriga y Hernández (2002) que hacen mención a tres maneras para conseguir los cambios de actitudes: la primera es proporcionando un mensaje convincente. La segunda, es proporcionar un modelaje de actitudes y la tercera es inducir una especie de conflicto entre los componentes cognitivos, afectivos y conductuales.

\section{CONCLUSIÓN O CONSIDERACIONES FINALES}

1) No existe influencia de los hábitos ecológicos en la conservación del medio ambiente en los alumnos del tercer grado de la institución educativa $\mathrm{N}^{\circ} 81011$ "Antonio Raimondi” de Trujillo en el año 2020, por cuanto el coeficiente rho de Spearman, arroja un valor para r igual a -,089 y un nivel de significancia p de ,526; cómo el Valor $\mathrm{p}>0.05$, en consecuencia, se acepta la hipótesis nula.

2) El nivel de hábitos ecológicos de los alumnos del tercer grado del nivel primario de la institución educativa $N^{\circ} 81011$ “Antonio Raimondi” de Trujillo en el año 2020 es, Logrado con $77,4 \%$, en proceso $22,6 \%$ y el $8 \%$ en inicio.

3) El nivel de conservación del medio ambiente de los alumnos del tercer grado de primaria de la institución educativa $\mathrm{N}^{\circ} 81011$ “Antonio Raimondi” de Trujillo en el año 2020 es, Logrado con 77,4\%, en proceso $22,6 \%$ y el $8 \%$ en inicio.

4) Con respecto a la relación de las dimensiones de la variable hábito ecológico con las dimensiones de la variable conservación del medio ambiente, se obtiene que no existe correlación en nueve de ellas, sin embargo, es necesario mencionar que existen tres relaciones débiles: la dimensión cognitiva con el reciclaje de residuos sólidos, la 
dimensión conativa con la limpieza del aula y la dimensión activa con el reciclaje de residuos sólidos.

\section{LISTA DE REFERENCIAS}

Barker, R. (1968). Ecological Psychology: Concepts and methods for studying the environment of human behavior. Palo Alto, CA. Estados Unidos: Stanford University Press.

Bazán, T. y Sotero, J. (2006) La formación ambiental. En la educación ambiental en la escuela. Guía de Estudio. Ciudad de México. México: SEP.

Berenguer, J. y Martín, R. (2003). Una aproximación al concepto de actitud ambiental. En C. San Juan, J. Berenguer, J.A. Corraliza e I. Olaizola (Eds.). Medio ambiente y participación, una perspectiva desde la Psicología Ambiental y el derecho. (pp. 55-64) Bilbao. España: Servicio editorial de la Universidad del País Vasco.

Capistrán, F. (1998). Taller sobre reciclaje de basura. Curso taller de Educación Ambiental. Ciudad de México. México: Xalapa.

Carabias, J. (1999). El deterioro ambiental: cambios nacionales, cambios globales. La educación ambiental en la escuela secundaria. Ciudad de México.México: SEP.

Chiape, G. (2016). Aprendizaje de Hábitos ecológicos en la conservación del agua y suelo en los niños de primer año de educación básica Juan Gutenberg Machachi. (Tesis doctoral inédita). Universidad Central del Ecuador. Ecuador.

Corraliza, J (2004). La investigación de la conciencia ambiental: Un enfoque psicosocial. Recuperado de https://www.juntadeandalucia.es/medioambiente/web/Bloques_Tematicos/Publi caciones_Divulgacion_Y_Noticias/Documentos_Tecnicos/personas_sociedad_y _ma/cap7.pdf

Corraliza, J. A. y Berenguer, J. (2000). Environmental values, beliefs and actions: A situational approach. Environment and Behavior, 32, 832-848. https://doi.org/10.1177/00139160021972829

Díaz Barriga, F. y Hernández, G. (2002). Estrategias docentes para un aprendizaje significativo: una interpretación constructivista. Ciudad de México. México: Mc Graw Hill.

Fernández, R. (1992). Por una sociedad ecológica. Barcelona. España: Gustavo Gili. 
Gareca, M. y Villarpando, H. (2017) Impacto de las áreas verdes en el proceso de enseñanza aprendizaje. Revista Ciencia Tecnología e Innovación. Vol.14, n.15, pp. Recuperado

de http://www.scielo.org.bo/scielo.php?script=sci_abstract\&pid=S2225$87872017000100006 \& \operatorname{lng}=\mathrm{es} \& \mathrm{nrm}=$ iso

Grupo Entorno (2001). Ecología, desarrollo y solidaridad. Madrid. España: CCS.

Hernández, R., Fernández, C. y Baptista, M. (2010). Metodología de la investigación. Bogotá, Colombia: Mc Graw Hill S.A.

Larriaga, S. (2016). Esquema de una Propuesta de Programa Director para la mejora de hábitos ecológicos en una institución educativa primaria. (Tesis doctoral inédita). Universidad Jorge Tadeo Lozano de Bogotá. Colombia.

Lehman, P. (2004). Behavior analysis and environmental protection: Accomplishments and potential for more. Behavior and Social Issues, 13, 13-32. http://dx.doi.org/10.5210/bsi.v13i1.33

Márquez, A. 1998. ANDRAGOGÍA, Propuesta Política para una Cultura Democrática en educación superior. Ponencia presentada en el Primer Encuentro Nacional de Educación y Pensamiento 9 al 11 de julio de 1998 Santo Domingo, República Dominicana. Recuperado de http://ofdp_rd.tripod.com/encuentro/ponencias/amarquez.html

Morejón, A. (2006). Formación de la conciencia ambiental: Importancia de la ética ambiental y la educación ambiental en este proceso. Recuperado de http://biblioteca.filosofia.cu/php/export.php?format=htm\&id=2355\&view=1

Páramo, P., \& Gómez, F. (1997). Actitudes hacia el medio ambiente: su medición a partir de la teoría de facetas. Revista Latinoamericana de Psicología, 29(2). Obtenido de http://www.redalyc.org/resumen.oa?id=80529202

Pereda, S (2016). Creando Conciencia Ambiental en niños y adolescentes de la Institución Educativa La Fuente de Tocancipa para rescatar y preservar el ambiente que nos queda" (Tesis doctoral inédita). Universidad Nacional de Colombia. Colombia.

Ricardes, J. (2016). Programa de cuidado ambientalista para el desarrollo de hábitos ecológicos en los alumnos de una Escuela Fiscal Mixta. (Tesis doctoral inédita). Universidad San Francisco de Quito. Ecuador. 
Saegert, S. y Winkel, G. H. (1990). Environmental Psychology. Annual Review of Psychology, 41(1), 441-477. doi: doi:10.1146/annurev.ps.41.020190.002301

Torres Santomé, Jurjo (1998). "Las razones del curriculum integrado", en Globalización e interdisciplinariedad: el currículo integrado. Madrid: Morata, pp. 29- 95. Recuperado de https://www.uv.mx/dgdaie/files/2012/11/CPP-DC-TorresSantome-Las-razones-del-curriculum.pdf

Vílchez, T. (2003). Educación ambiental, conceptos y propuestas. Madrid. España: Consejo Central Social. 